\title{
Berichtigungen zu den Beiträgen der Flora von Salzburg.
}

(Oesterr, botanische Zeitschrift 1862. 12. Bd. Seite 305.)

Von Julius Hinterhuber.

Thalictrum simplex L. Wurde im Nassfelde von Mielichh ofer und nicht von Dr. Sauter anfgefunden.

Anemone baldensis L. Kommt auf den Fuschertauern nicht vor, sondern wurde von Dr. S a u ter ober der Ringalpe in der Fusch, und in der Zwing in Pinzgau gefunden.

Ranunculus alpinus L. Soll bei Maria Ellenberg in Schellenberg $\mathrm{nicht}$ Schallenberg heissen.

R. arvensis L. Kommt bei Wals bei Salzburg vor.

R. pantothrix DC. Allgemein in Lungau verbreitet Dr. Sauter.

R. platanifolius L. Ist nur eine Form von Ranunculus aconitifolius.

R. pygmaeus Wahlb. Wurde in der Zwing in Pinzgau von Herrn B a mberger aufgefunden.

R. Traunfellneri Hoppe. Dieser von Herrn Jelmelli auf der übergossenen Alpe aufgefundene, ist nicht der wahre, sondern nur eine Form von $R$. alpestris $\mathrm{L}$.

Helleborus foetidus L. Wurde bei Mattsee nicht von Dr. Sauter, sondern von Herrn Pfarrer Michl aufgefunden.

Aequilegia Einseliana Schultz. Ist nur eine Form von Aquilegia pyrenaica DC.

Delphinium elatum L. Wurde auf dem Scheidberg von Herrn Pfarrer Michl und nicht von Dr. Sa uter aufgefunden.

Fumaria Vaillantii Lo is. Kommt bei Salzburg in Gürten und nicht in Gräben vor.

Barbarea stricta. Andrz. Kommt nach Dr. Sauter auf feuchten Wiesen bei Leopoldskron vor.

Arabis Turrita L. Ist auf dem Neuhauserberge bei Salzburg nach Dr. Sauter zu streichen.

Draba tomentosa W ahlb. Soll heissen auf dem Eggerfürst nicht Churfürst. Ueberhaupt wurde der Standort Eggerfürst mehrfach mit Churfürst verwechselt.

Thlaspi montanum L. Soll heissen auf Aeckern in Lungau. Mielich hoffer.

Subularia aquatica L. Vor mehreren Jahren von Braune in einer Lache bei Grödig entdeckt. Wurde aber neuerer Zeit nicht mehr aufgefunden.

Aethionema saxatilis R. Br. Kommt auf dem Kies des Rosittenbaches, am Fusse des Untersberges vor. Dr. Sauter.

Viola palustris L. Auf den Torfmooren bei Salzburg nicht selten. Dr. Sauter. 
Dianthus Armeria L. Soll bei Eugendorf heissen.

Silene excapa All. Ist nur eine Form von Silene acaulis $\mathrm{L}$.

Facchinia lanceolata Rb. Soll auf der Enskrachse bei Radstadt nicht Rudkrachse heissen.

Arenaria Marschlinsii, wurde nicht von Dr. Sauter, sondern von Apotheker Traunsteiner aufgefunden.

Elatine triandra. Soll heissen a m nicht in Zellersee.

Hypericum veronense $\mathrm{Sch} r$. Ist nur eine Form von $H$. perforatum

L. Diese Pflanze wurde auch von Dr. Sauter bei Golling hinter der Kirche aufgefunden.

Trifolium glareosum Sch. Kommt nach Dr. Sauter allgemein auf Schutt von Gebirgsbächen vor.

Vicia dasicarpa Ten. Ist nur eine Form von Vicia glabrescens Koch, und kommt nach Dr. Sauter nicht selten in Getreidefeldern um Salzburg vor.

Vicia pisiformis, ist nach Dr. Sauter aus der Flora von Salzburg zu streichen.

Potentilla nerwegica L. Kommt nach Dr. Sauter bei Lambach in Pinzgau vor.

P. salisburgensis. Soll Eggerfürst bei Hallein, nicht Churfürst bei Hallein heissen.

Alchemilla fissa. Soll ebenfalls Eggerfürst bei Hallein, nicht Churfürst bei Hallein heissen.

Cotoneaster tomentosa Lindl. Kommt auf einer Felswand auf dem Mönchberg bei Salzburg, nicht auf einer alten Mauer vor.

Sempervivum arenarium Koch. Ist nur eine Form von Sempervivum hirtum $\mathrm{L}$.

S. Funkii Braune. Wurde der Standort sicherlich mil Sempervioum Wulfenii $\mathrm{H}$ oppe verwechselt.

Saxifraga Facchinii Koch. Wurde auf dem Preber in Lungau von Vierhapper aufgefunden.

S. retusa Stbg. Wurde aufgefunden in der Fusch auf dem Schwarzkopf von Herrn Hofgärtner Schmid; auf dem Hoch-Golling in Lungau von Dr. Sauter.

Helosciadium repens Koch. Kommt nach Dr. Sauter auch bei Glas vor.

Aster parviflorus N. E. und Aster salignus Willd, sind aus der Flora zu streichen, und dafür $\boldsymbol{A}$. bellidiflorus in den Auen an der Salzach bei Weitwörthen zu setzen. Dr. Sauter.

Carpesium cernuum L. An einem Hügel bei Laufen, nicht in den Auen an der Salzach. Birnbacher Curat.

Artemisia campestris L. Um Salzburg. Dr. Sauter.

Achillea Clusiana T a u ch. Auf dem Radstädter-Tauern. Hille br and. Senecio alpinus K. Auf dem Radstädter-Tauern. Z wanziger.

$S$. incanus L. Ist synonym mit $S$. carniolicus Willd.

$S$. nebrodensis $\mathrm{L}$. Ist synonym mit $S$. rupestris Kit.

Carlina longifolia $\mathrm{R} b$. Kommt nach Metzler auch am westlichen

Gehänge von Böckstein vor. 
Picris crepoides Saut. Ist nur eine Form von P. hieracioides. Leontodon nigricans $\mathrm{K}$ it. und Tarxacum nigricans sind synonym. Hieracium pilosellaeforme $\mathrm{Hoppe}$ ist synonym mit $H$. Hoppeanum Sch. Auf Bergmäder ober dem Bade Fusch. Dr. Sauter. $\boldsymbol{H}$. rigidum W. ist zu streichen, dafür aber an dessen Stelle $\boldsymbol{H}$. nemorosum Pers. anzusetzen.

Campanula thyrsoidea L. Auf einer Alpe im Mühlbachthale, ober Keprun in Ober-Pinzgau. Dr. Sauter.

Swertia perennis L. Auf dem Moore unter der Baderluck gegen Fuschel zu. Dr. Sauter.

Linaria Cymbalaria $\mathrm{L}$. An benanntem Orte verwildert. Dr. Sa uter. Orobanche neotioides Saut. Am Gangsteige von St. Gilgen nach

Feistenau im Walde, wie auch bei Abtenau. Dr. Sauter.

Or. pallidiflora Wimm. Ist synonym mit 0 . Galii. Dr. Sauter.

Or. procera K. Ist synonym mit $O$. erubescens. Dr. Sa uter.

Or. Sauteri. Ist synonym mit 0 . Cardui. Dr. Sauter.

Galeopsis grandiflora Roth. Ist synonym mit G. ochrolenca Lam. Lysimachia thyrsiflora $L$. Bei Kasham neben der Strasse nach

Pöllheim in einem Sumpfe. Dr. Sauter.

Anagallis tenella L. Bei Saalfelden zu streichen. Dr. Sauter. Primula truncata $\mathrm{S} \mathrm{ch}$. Ist eine Form von Pr. minima $L$.

P. tyrolensis Schultz. Auf dem Tannengebirge Mielichh. Auf dem Bischof neben dem Hallstädter Schneeberge. Fe hr e $\mathrm{n} b$ a ch. Rumex obtusifolius L. Allenthalben in Salzburg gemein. Dr. S a u ter. Potamogeton filiformis Pers. In einem künstlichen Sumpfe bei

Abtenau. Neuerer Zeit nicht mehr aufgefunden. Dr. Sauter. Najas major L. In Wassergräben bei Salzburghofen. Lemna polyrrhiza L. In einer Lache bei Köttellack. Allium montanum $\mathrm{S}$ chmd. Ist synonym mit $\boldsymbol{A}$. fallax Don. Carex maxima S cop. Ist synonym mit $C$. pendula Schreb. C. Personii Sieb. Ist synonym mit C. Gebhardi Hoppe.

Salzburg, den 7. April 1863.

\section{Correspondenz.}

Bihar bei Grosswardein, den 10. September 1863.

Seit ich ihnen das letzte Mal schrieb, bin ich, und zwar seit 29. Juli hier in Bihar, nicht ganz 2 Meilen von Grosswardein, wohin ich auf einem kürzeren Wege reitend, in 1 Stunde gelangen kann. Bei mir besitze ich nur ganz kleine Partien meines Herbars, nämlich solche Gattungen, die ich fort und fort studiere, wie: Sesleria, Colchicum, Iris, Muscari, Trifolium etc. etc. - Doch reite ich beinahe täglich nach Grosswardein, um irgend im Herbar oder in Büchern etwas über Pflanzen, die mir gerade einfallen, nachzusehen. Die wichtigeren Floren 